\title{
Vitamin A supplementation in developing countries
}

Children of poor families in less developed countries all too often succumb to a downward spiral of malnutrition and infection. However, recent research, showing that vitamin A supplementation may break open this vicious cycle and reduce childhood mortality, has generated considerable excitement. Studies in Indonesia established a clear link between vitamin A status and child mortality. ${ }^{1}$ Work in other countries then confirmed that vitamin A supplements can decrease mortality by an average of $23 \%$ among children aged 6 months to 5-7 years in communities where xerophthalmia, that is clinical vitamin A deficiency, is prevalent. ${ }^{2}$ Several forms of supplements, for example periodic large dose capsules, weekly low doses of oil, or fortified food, appear equally effective. Differences in the impact of different supplementation trials may result partly from differences in the most frequent causes of death within the study population as vitamin A appears to exert its greatest benefits on morbidity and mortality from measles and diarrhoea. ${ }^{2-4}$ There are several important unresolved questions concerning vitamin A supplementation. Firstly, there is very little information about effects on children outside the 6 months to 5 year age range and it is not clear whether supplements are safe or efficacious when given to younger infants. Secondly, all studies have been conducted in areas where xerophthalmia was prevalent, even though children with clinical signs of vitamin A deficiency were generally excluded from randomised trials for ethical reasons. The impact of supplementation on populations with subclinical vitamin A deficiency without xerophthalmia merits attention.

\section{Biological basis on which to make decisions about supplementation}

The decision as to which populations or individuals would be most likely to benefit from vitamin A supplements should be based on two main considerations: the degree of vitamin A deficiency present and the expected protective functions of vitamin A. Unfortunately, there remain large gaps in our knowledge of both these points. Xerophthalmia is a very insensitive measure of vitamin A status. Conjunctival impression cytology is advocated by some investigators as a more sensitive means of assessing the eye damage of vitamin A deficiency ${ }^{5}$ but others have found that samples are hard to obtain from young children, that agreement on the microscopical findings is often not achieved and that the results bear little or no relation to other measures of vitamin A status and do not change after supplementation. ${ }^{67}$ Serum retinol is a more sensitive indicator of poor vitamin A status but lacks specificity since it decreases as part of the generalised acute phase response to infection or trauma. ${ }^{8}$ It is unclear whether or not this decrease results in a temporary functional vitamin A deficiency even in individuals with apparently adequate liver retinol stores. It is clear, however, that the decrease in plasma retinol will complicate assessment of vitamin A status in the patients and communities whose status is of most concern. The relative dose response (RDR) and modified RDR tests are designed to measure liver retinol stores which are more sensitive to deficiency than is serum retinol. Although these tests show promise, preliminary results suggest the RDR is affected by malabsorption and by systemic infection which decreases release of retinol binding protein, a negative acute phase protein, from the liver. ${ }^{9}$ Since vitamin $\mathrm{A}$ is concentrated in relatively few foods, for example green leafy vegetables or yellow and orange fruits and vegetables, food frequency questionnaires to determine habitual intake can relatively quickly and cheaply provide a reasonable estimate of whether supplementation or other means of improving vitamin A intake of a population is likely to be necessary. ${ }^{10}$

Knowing the biological actions of vitamin A supplements would be a help when determining which patients presenting at clinics and hospitals might benefit. Vitamin A supplements are now recommended for children hospitalised with measles in any area where either xerophthalmia is seen or where the measles case fatality rate is greater than $1 \% .{ }^{11}$ As even American hospitals may experience a measles case fatality rate greater than $1 \%, 12$ it appears that the recommendation is for universal treatment of measles with vitamin A. There are, as yet, virtually no published data on the effectiveness of treating other infections with vitamin A. A recent epidemiological study, however, showed that malaria parasitaemia and deaths from malaria were uninfluenced by supplements. ${ }^{13}$ The interaction of vitamin A and HIV infection is of interest; mothers with low serum retinol have higher rates of HIV transmission to their infants. ${ }^{14}$

\section{Methods of improving vitamin A status}

The long term solution to the problem of poor vitamin A status is to increase intake of vitamin A rich foods. This dietary solution has the virtues of being relatively cheap, since vitamin $\mathrm{A}$, unlike another important micronutrient, iron, is found in low cost fruits and vegetables, and of being likely to improve status of the whole population, not just particular target groups such as children. An increase in the consumption of vitamin A rich foods will require social marketing, ${ }^{15}$ improved processing and preservation of foods to maintain carotene concentrations, and greater recognition of what vitamin A rich foods are locally available and acceptable, especially to young children.

Vitamin A fortification of foods has been tested; for example fortification of monosodium glutamate improved vitamin A status and decreased mortality among children in Indonesia. ${ }^{16}$

As changing dietary habits requires time and since fortification may be administratively complicated, discussions about improving vitamin A status eventually tend to return to the use of periodic high dose capsules. A capsule containing $60 \mathrm{mg}$ (200000 IU) of retinol given every four months appears adequate to improve vitamin A status and decrease mortality of children 1-5 years old. Children 6-12 months old can similarly be supplemented with $30 \mathrm{mg}$ retinol. The most cost effective means of getting these capsules to the children at risk of deficiency is unclear. Thrice yearly public health campaigns would be expensive and motivation is unlikely to be sustainable. Capsules can be given to children who present at hospitals or clinics but, in general, it would be preferable to provide children with vitamin A before, rather than after, they become ill. Recently there has been intense debate concerning the pros and cons of providing vitamin A capsules to children coming for the Expanded Programme on Immunization (EPI) vaccinations. A major advantage of this approach is its low cost as no additional contacts with children, requiring additional personnel, are necessary. A secondary benefit is that, since vitamin A in large doses is a known adjuvant, ${ }^{17}$ giving capsules at the time of vaccination may increase the response to the vaccine, 
although further research is needed. However, strong resistance to the joining of vitamin A supplementation to EPI vaccinations has also been registered, mainly because there is some evidence of toxicity. In Bangladeshi children, 15 mg (50000 IU) retinol given with diphtheria, pertussis, and tetanus vaccines resulted in an $11 \%$ excess incidence of bulging fontanelle in the subsequent two days. ${ }^{18}$ The medical significance of bulging fontanelle is disputed but, even if it is harmless, parental concern about the symptom could jeopardise both the supplementation and the vaccination programmes. Thus caution is required before implementing this approach widely.

There are additional reasons for caution about including vitamin A capsules with EPI vaccines. Vaccines are given at about 6, 10 and 14 weeks for diphtheria, pertussis, and tetanus and 9 months for measles. However, as breast feeding is extremely effective at preventing vitamin A deficiency, 1920 infants at these ages are not the most at risk population for vitamin A deficiency. Furthermore, in young breast fed infants, respiratory diseases are a far more important cause of morbidity and mortality than gastrointestinal diseases whereas vitamin A supplements appear to protect primarily against gastrointestinal illness. Therefore, there is still debate whether young infants need or should be given large doses of vitamin A, whether with or without vaccination.

Promotion of breast feeding is an important component of any programme to improve vitamin A status. The quantity of the vitamin delivered to the infant through breast milk can be increased by giving high dose capsules to mothers within four weeks of giving birth when there is no danger of their being pregnant and thus no danger of the teratogenic effects of vitamin A. A single postpartum supplement to the mother can increase her milk retinol concentration for at least eight months and infant vitamin A stores at 6 months. ${ }^{20}$ Breast feeding and postpartum supplementation should be advocated strongly since they are safe, cheap, and an effective means of improving vitamin A status.

Vitamin A status may also be improved by decreasing the prevalence of infection. Prolonged or severe diarrhoea may impair absorption of the vitamin and certain infections, particularly those which damage epithelia, may result in depletion of retinol stores. ${ }^{2122}$ Thus, successful immunisation against measles has a high priority and it would also be interesting to determine the effects on vitamin A status of deworming or of impregnated bed nets for prevention of malaria.

\section{Conclusions}

That adequate vitamin A status is an important component of protection against childhood infectious disease mortality in many developing countries is now well established. There remains considerable scope for improving practical approaches by which to promote vitamin A consumption. Cooperation between basic scientists, medical workers, public health administrators, and educators could have a major impact on child health.

\section{SUZANNE $M$ FILTEAU}

Centre for International Child Health, ANDREW M TOMKINS

Institute of Child Health,

30 Guilford Street,

London WC1N $1 E H$

1 West KP, Howard GR, Sommer A. Vitamin A and infection: public health implications. Annu Rev Nutr 1989; 9: 63-86.

2 Beaton GH, Martorell R, Aronson KJ, et al. Effectiveness of vitamin A supplementation in the control of young child morbidity and mortality in developing mentation in the control of young child
countries. Geneva: ACC/SCN, 1993.

3 Ghana VAST Study Team. Vitamin A supplementation in northern Ghana: effects on clinic attendances, hospital admissions, and child mortality. Lancet 1993; 342: 7-12.

4 Fawzi WW, Herrera MG, Willett WC, Nestel P. Dietary vitamin A intake and the risk of mortality among children. Am $\mathcal{F}$ Clin Nutr 1994; 59: 401-8. 5 Carlier C, Etchepare M, Ceccon J-F, Amedee-Manesme O. Assessment of the vitamin A status of preschool and school age Senegalese children during a cross-sectional study. Int $\mathcal{f}$ Vitam Nutr Res 1992; 62: 209-15.

6 Nathanail L, Powers HJ. Vitamin A status of young Gambian children: biochemical evaluation and conjunctival impression cytology. Ann Trop Paediatr 1992; 12: 67-73.

7 Ross DA, Badu JK, Amidini A, et al. A comparison of serum retinol levels and conjunctival impression cytology results in young children in Ghana. Proceedings of the XV Meeting of the International Vitamin A Consultative Proceedings of the XV Meeting of
Group. Arusha, Tanzania, 1993.

8 Filteau SM, Morris SS, Abbott RA, et al. Influence of morbidity on serum retinol of children in a community-based study in northern Ghana. $A m \mathcal{F}$ Clin Nutr 1993; 58: 192-7.

9 Hussey G, Sive A, Mallan H, Dempster W. The vitamin A status of children with acute infections: as assessed by the relative dose response test. Proceedings of the XV Meeting of the International Vitamin $A$ Consultative Group. Arusha, Tanzania, 1993: abstract.

10. International Vitamin A Consultative Group. Guidelines for the development of a simplified dietary assessment to identify groups at risk for inadequate intake of vitamin $A$. Washington: IVACG, 1989 .

11 World Health Organisation. Joint WHO/Unicef statement on vitamin A for measles Wkly Epidemiol Rec 1987; 62: 133-4.

12 Butler JC, Havens PL, Sowell AL, et al. Measles severity and serum retinol (vitamin A) concentration among children in the United States. Pediatrics 1993; 91: 1176-81.

13 Binka FN, Ross DA, Morris SS, et al. Vitamin A supplementation and childhood malaria in northern Ghana. Am $\mathfrak{f}$ Clin Nutr (in press).

14 Semba RD, Miotti PG, Chiphangwi JD, et al. Maternal vitamin A deficiency and mother-to-child transmission of HIV-1. Lancet 1994; 343: 1593-7.

15 Smitasiri S, Attig GA, Dhanamitta S, Tontisirin K. Social marketing for vitamin A-rich foods in Thailand. Nakhon Pathom: Institute of Nutrition, vitamin A-rich foods in Thaila

16 Muhilal, Permeisih D, Idjradinata YR, Muherdiyantiningshi, Karyadi D. Vitamin A-fortified monosodium glutamate and health, growth, and survival of children: a controlled field trial. Am $f$ Clin Nutr 1988; 48: 1271-6.

17 Ross AC. Vitamin A status: relationship to immunity and the antibody response. Proc Soc Exp Biol Med 1992; 200: 303-20.

18 De Francisco A, Chakrabory J, Chowdhury HR, et al. Acute toxicity of vitamin A given with vaccines in infancy. Lancet 1993; 342: 526-7.

19 Newman V. Vitamin A and breastfeeding: a comparison of data from developed and developing countries. San Diego: Wellstart, 1993.

20 Stoltzfus RJ, Hakimi M, Miller KW, et al. High dose vitamin A supplementation of breast-feeding Indonesian mothers: effects of the vitamin A status of mother and infant. $¥$ Nutr 1993; 123: 666-75.

21 Campos FACS, Flores H, Underwood BA. Effect of an infection on vitamin A status of children as measured by the relative dose response. Am $\mathfrak{f}$ Clin A status of children as

22 West CE, Sijtsma SR, Kouwenhoven B, Rombout JHWM, van der Zijpp AJ. Epithelia-damaging virus infections affect vitamin $A$ status in chickens. F Nutr 1992; 122: 333-9.

\section{Minimal access surgery in paediatrics}

Wickham noted that three seminal events have indelibly altered surgery: the introduction of anaesthesia, the development of antiseptics, and endoscopy. ${ }^{1}$

Minimal access surgery (MAS) or 'endoscopic surgery' is the execution of established surgical procedures in a way that leads to a reduction of the trauma of access. Surgical procedures are conducted by remote manipulation within the close confines of body cavities or lumen of hollow organs under visual control via telescopes and television screens. Endoluminal surgery (bronchoscopy, gastro- intestinal endoscopy, endourology, and lithotripsy), an important part of MAS, is already well established and is not discussed further.

\section{History}

The idea of MAS is not new; the use of tubes in medicine dates from the earliest days of civilisation in Mesopotamia and ancient Greece. Modern endoscopy started in 1805 when Bozzini, an obstetrician from Frankfurt, using 